\title{
Nanobiotechnology and nanomedicine: small change brings big difference
}

\author{
Shizhu Chen ${ }^{1,2} \&$ Xing-Jie Liang ${ }^{1,3^{*}}$ \\ ${ }^{1}$ CAS Key Laboratory for Biomedical Effects of Nanomaterials and Nanosafety, CAS Center for Excellence in Nanoscience, National Center for \\ Nanoscience and Technology of China, Beijing 100190, China; \\ ${ }^{2}$ College of Chemistry \& Environmental Science, Chemical Biology Key Laboratory of Hebei Province, Key Laboratory of Medicinal Chemistry \\ and Molecular Diagnosis of Ministry of Education, Hebei University, Baoding 071000, China; \\ ${ }^{3}$ University of Chinese Academy of Sciences, Beijing 100049, China
}

Received March 1, 2018; published online April 1, 2018

Citation: Chen, S., and Liang, X.J. (2018). Nanobiotechnology and nanomedicine: small change brings big difference. Sci China Life Sci 61, 371-372. https:// doi.org/10.1007/s11427-018-9293-2

The physicist Richard Feynman put forward the concept of nanotechnology in his famous speech, "There's Plenty of Room at the Bottom," at the annual meeting of the American Physical Society on December 29, 1959. He began with a "bottom up" approach and proposed assembling from a single molecule or even an atom to meet the requirements of the design. He indicated that the laws of physics do not rule out the possibility of making an object by atom stack and predicted that our precise control over tiny objects could significantly expand the scope of material attributes acquired by human beings. Furthermore, in his report published in 1960, Richard Feynman proposed the concept of nanosurgery and nanodrug delivery devices for the first time and further predicted that nanotechnology will play an important role in the field of life and health sciences in the future (Feynman, 1960).

Since the time the abovementioned concept was proposed, pharmaceutical researchers began gradually accepting the connotation of nanomedicine. The breakthrough of nanomedicine depends primarily on the development of its carrier technology, which is still in the progress of nanotechnology in general (Kim et al., 2010). Since the past few decades, nanomedicine has been continuously contributing toward innovations in the field of therapeutics, especially in the

*Corresponding author (email: liangxj@nanoctr.cn) therapeutic areas of antitumor, antiviral, hormonal, anti-inflammatory, and biological macromolecular drugs. Continuous investment support from governments and large pharmaceutical companies across the world could help in achieving significant progress in nanomedicine to develop novel technologies that could be approved for marketing and applied in clinical treatment. In the context of such a splendor, although there are several new technologies and methods, a large gap exists between the bench and the clinic, and even the nanomedicine on the market has few exciting clinical benefits, which is far away from people's expectation (Juliano, 2013; Venditto \& Szoka Jr, 2013). Isn't the soughtafter nanotechnology just a beautiful utopia? Therefore, we have to stop slowly to review nanomedicine and further try to determine the status of its research and development and applications, as well as to outline its future trend rationally.

ChinaNano has become an important platform for international nanotechnology exchanges, which has attracted wide attention all over the world. With strong support from the Ministry of Science and Technology, the Ministry of Education, and the Chinese Academy of Sciences, seven conferences have been successfully held, namely, ChinaNANO 2005, ChinaNANO 2007, ChinaNANO 2009, ChinaNANO 2011, ChinaNANO 2013, ChinaNano 2015, and ChinaNano2017, which have aroused a great response in the academic circles at home and abroad. At present, China- 
NANO has developed into a brand conference in the field of nanotechnology and as a bridge for communication between Chinese scientists and international academic communities. In this conference, nanobiotechnology and nanomedicine session was set up as usual to invite well-known scholars around the world to exchange and grasp the development trends in nanobiotechnology.

This thematic issue is dedicated to ChinaNano Nanobiotechnology and Nanomedicine session, and the contributors are renowned experts in the field of nanobiology. In this issue, we will be focusing on new applications of nanotechnology in the field of life and health sciences. Today, nanotechnology is not limited to the manufacture of an antitumor nanomedicine (nanodrug delivery system), whose applications have spread to other diseases such as obesity (Zhang et al., 2018) and diseases of the central nervous system (Wang \& Xue, 2018). It is also applied to the biological effects and safety evaluation of nanomaterials (Jin et al., 2018; Li et al., 2018), which remains as one of the major concerns of nanomedicine researchers. We believe that this issue could provide some new ideas for the future develop- ment of nanobiotechnology and nanomedicine.

Compliance and ethics The author(s) declare that they have no conflict of interest.

Feynman, R.P. (1960). There's plenty of room at the bottom. Eng Sci, 23, 22-36.

Jin, Y., Chen, S.Z., Li, N., Liu, Y.J., Cheng, G., Zhang, C.M., Wang, S.X., and Zhang, J.C. (2018). Defect-related luminescent bur-like hydroxyapatite microspheres induced apoptosis of MC3T3-E1 cells by lysosomal and mitochondrial pathways. Sci China Life Sci 61, 464-475.

Juliano, R. (2013). Nanomedicine: is the wave cresting? Nat Rev Drug Discov 12, 171-172.

Kim, B.Y.S., Rutka, J.T., and Chan, W.C.W. (2010). Nanomedicine. N Engl J Med 363, 2434-2443.

Li, Z., Persson, H., Adolfssom, K., Oredsson, S., and Prinz, C. (2018). Morphology of living cells cultured on nanowire arrays with varying nanowire densities and diameters. Sci China Life Sci 61, 427-435.

Venditto, V.J., and Szoka Jr., F.C. (2013). Cancer nanomedicines: So many papers and so few drugs! Adv Drug Deliver Rev 65, 80-88.

Wang, R., and Xue, X. (2018). Theranostical application of nanomedicine for treating central nervous system disorders. Sci China Life Sci 61, 392-399.

Zhang, Y.Q., Yu, J.C., Qiang, L., and Gu, Z. (2018). Nanomedicine for obesity treatment. Sci China Life Sci 61, 373-379.

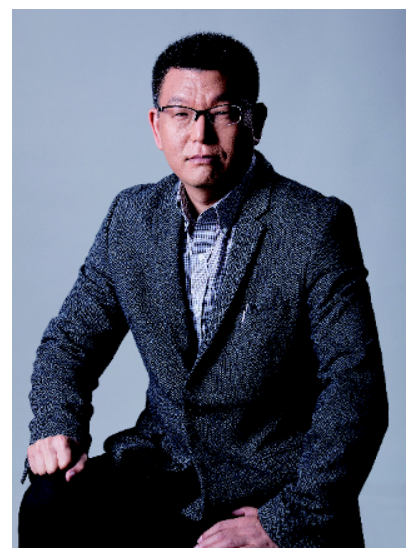

Biographical Sketch

Dr. Xing-Jie Liang obtained his Ph.D. at National Key Laboratory of Biomacromolecules, Institute of Biophysics, Chinese Academy of Sciences. He finished his postdoc with Dr. Michael M. Gottesman at Laboratory of Cell Biology, Center for Cancer Research, National Cancer Institute, National Institutes of Health. Then, he worked as a Research Fellow at Surgical Neurology Branch, NINDS (National Institute of Neurological Diseases and Strokes, NIH) for 2 years. In 2007, he was an Assistant Professor at Department of Radiology, School of Medicine, Howard University. Dr. Liang currently is deputy director of Key Laboratory for Biomedical Effects of Nanomaterials and Nanosafety, Chinese Academy of Sciences and a Principal Investigator at National Center for Nanoscience and Technology of China. Dr. Liang is a founder member of International Society of Nanomedicine, member of American Association for Cancer Research, and member of American Society of Cell Biology. His research interests are in elucidating mechanisms to improve nanomedicinal bioavailability by nanotechnology in vitro and in vivo, and novel strategies to increase therapeutic effect on cancers and infective diseases. 